\title{
Study on Deformation Behavior of Ti6Al4V Parts Fabricated by Selective Laser Melting
}

\author{
Tao Yang ${ }^{1}$, Tingting Liu ${ }^{1}$, Wenhe Liao ${ }^{1, a}$, Changdong Zhang ${ }^{1}$, Shengqin Duan ${ }^{1}$ and Xiangyuan Chen ${ }^{1}$ \\ ${ }^{1}$ School of Mechanical Engineering, Nanjing University of Science and Technology, Nanjing, Jiangsu 210094,China
}

\begin{abstract}
During the Selective laser melting process, the accumulation of the residual stress caused by the excessive temperature gradients and the rapid solidification is the main factor causing deformation; and the wetting quality of the meltpool also has an impact. How to control the deformation is the key problem to improve the quality of the parts. In this paper, the thin-wall specimens are divided into four distinct conditions, analyzing the meltpool date collected by the melting pool monitoring system and the simulation of temperature field, the relationship between the process parameters and the deformation under the same energy density is discussed. The results show that the temperature, the width and the intensity of the meltpool increase with the increasing of the laser power and the scanning speed, but the temperature gradients and the rate of the solidification of the meltpool are on the contrary, resulting in the decrease of the residual stress and the deformation of the part, which is help to improve the quality of the part.
\end{abstract}

\section{Introduce}

Titanium and associated alloys are favored by the aerospace field due to their low density, high strength, high temperature resistance and strong compatibility [1]. However, the parts are difficult cutting because of the high hardness, because it is costly to use traditional processing methods. Selective Laser Melting (SLM), a form of powder bed fusion (PBF) as defined in the standard terminology described in the ISO/ASTM 52900 standard, is a promising method that has the advantages of manufacturing flexibility, complex shape design, time and cost savings [2]. Forming the titanium alloy parts, especially the parts with complex geometric shapes, has obvious advantage. In recent years, it has received extensive attention and has a wide range of application in aerospace, biomedical and other fields [3].

However, the thermal stress is easily generated in the forming process due to the characteristics of melting fast and cooling fast in the SLM, which may cause the deformation of the parts and even cracks [4], and the defects have a significant influence on the dimensional accuracy and mechanical properties of the parts [5]. The Fraunhofer Institute of Laser Technology [6] found that the rate of temperature variation in the process has a great influence on the surface quality and $p$ mechanical properties of the fabricated parts. Buchbinder et al. [7] and SiddiqueS et al. [8] studied that preheating the substrate may suppress cracks and reduce deformation effectively due to reduce the temperature

\footnotetext{
a Corresponding author : cnwho@njust.edu.cn
} 
gradient. Karg et al. [9] found that reducing the cooling rate of the molten pool can reduce the susceptibility of Al-Cu alloys to cracks during SLM process.

The above results show that change the temperature field can control the solidification rate of the molten pool and the cooling rate of the specimen in the process Select a suitable temperature field can reduce the deformation of the specimens and improve the quality. In this paper, combining the data of the molten pool and the results of the experiment, with the help of the temperature field simulation, analyzing the influence of the process parameters on the solidification rate of the molten pool and the cooling rate of the specimen. The relationship between the solidification rate, cooling rate and the residual stress are investigated, in order to provide guidance for optimizing the process parameters and improving the quality of the titanium alloy parts fabricated by SLM.

\section{Experiment method}

\subsection{Material and Equipment}

The powder used in this experiment was gas atomized Ti6Al4V supplied by FALCONTECH (WuXi), The chemical composition is shown in Table 1 . The Ti6Al4V powders were spherically shaped with a spheroidization ratio of almost $100 \%$ as seen in Fig.1(a). Granulometric parameters of the Ti6Al4V powders were evaluated by a laser particle size analyzer (Mastersize 3000), the D10, D50 and D90 of the sieved powder were $22.1 \mu \mathrm{m}, 33.5 \mu \mathrm{m}$ and $50.1 \mu \mathrm{m}$ respectively as seen in Fig.1(b).

Table 1. Chemical component of Ti6Al4V powders (atomic fraction, \%).

\begin{tabular}{|c|c|c|c|c|c|c|c|c|c|c|c|c|c|}
\hline $\mathbf{A l}$ & $\mathbf{V}$ & $\mathbf{F e}$ & $\mathbf{C}$ & $\mathbf{O}$ & $\mathbf{N}$ & $\mathbf{H}$ & $\mathbf{M o}$ & $\mathbf{M n}$ & $\mathbf{C u}$ & $\mathbf{S n}$ & $\mathbf{Y}$ & $\mathbf{Z r}$ & $\mathbf{T i}$ \\
\hline $5.5 \sim 6.75$ & $3.5 \sim 4.5$ & 0.14 & 0.007 & 0.13 & 0.007 & 0.002 & $\begin{array}{c}< \\
0.05\end{array}$ & $\begin{array}{c}< \\
0.05\end{array}$ & $\begin{array}{c}< \\
0.05\end{array}$ & $\begin{array}{c}< \\
0.05\end{array}$ & $\begin{array}{c}< \\
0.05\end{array}$ & $\begin{array}{c}< \\
0.05\end{array}$ & Bal \\
\hline
\end{tabular}
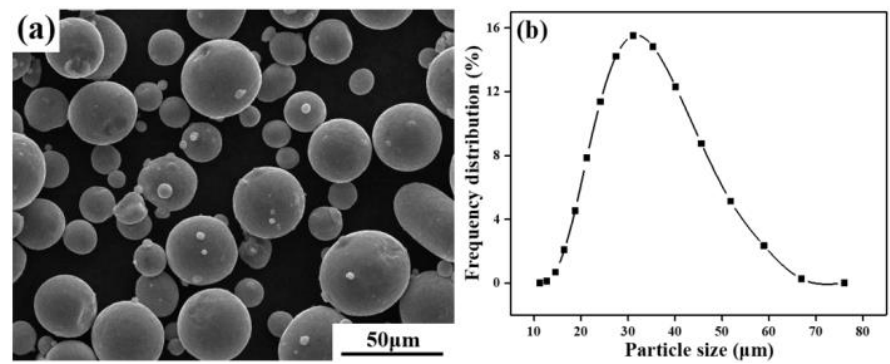

Figure 1. Typical particle morphology and particle size distribution curve of the Ti6Al4V powder: (a) SEM image of AlSi10Mg powder and (b) particle size distribution curve.

All specimens were fabricated using a Concept Laser M2. The M2 has an Yb-fiber laser with a laser power providing up to $400 \mathrm{~W}$ with a $60 \mu \mathrm{m}$ laser spot size, the wave length range is $1064-1100$ $\mathrm{nm}$. All process were completed in an Argon atmosphere with an oxygen content below $0.1 \%$ [10].

\subsection{Design of experiments}

In this paper, four groups of experiments were designed to study the influence of laser power and scanning speed on the solidification time of the molten pool and the cooling time of the parts as the same linear energy density. The process parameters are shown in Table 2. The linear energy density (E) has been calculated using Eq. (1) describing the energy input per unit length during SLM, and the E is combination of effects of the SLM process parameters.

$$
E=\frac{P}{V}
$$

where $\mathrm{P}(\mathrm{W})$ denotes the laser power, $\mathrm{V}(\mathrm{mm} / \mathrm{s})$ is the scanning speed. 
Table 2. The process parameters of the specimens.

\begin{tabular}{|c|c|c|c|c|c|}
\hline Number & $\begin{array}{c}\text { Power } \\
\mathbf{P}(\mathbf{W})\end{array}$ & $\begin{array}{c}\text { Velocity } \\
\mathbf{v}(\mathbf{m m} / \mathbf{s})\end{array}$ & $\begin{array}{c}\text { Scan } \\
\text { strategy }\end{array}$ & $\begin{array}{c}\text { Layer } \\
\text { thickness, } \\
\mathbf{t}(\boldsymbol{\mu} \mathbf{m})\end{array}$ & $\begin{array}{c}\text { Linear } \\
\mathbf{e n e r g y ~} \mathbf{E} \\
(\mathbf{J} / \mathbf{c m})\end{array}$ \\
\hline 1 & 120 & 1050 & \multirow{2}{*}{ Single } & 30 & \multirow{2}{*}{114.3} \\
\hline 3 & 180 & 1575 & & \\
\hline 4 & 250 & 2188 & & \\
\hline
\end{tabular}

In order to avoid the influence of the shape on the result, three different kinds of shape were designed such as circle, equilateral triangle and square, shown as Fig.2. The length of the square and the equilateral triangle is $10 \mathrm{~mm}$, and the diameter of the circular specimen is also $10 \mathrm{~mm}$, the wall thickness of all the specimens is $0.1 \mathrm{~mm}$, the height is $5 \mathrm{~mm}$. The specimens were scanned once and the laser scanning path is shown in Fig.3.

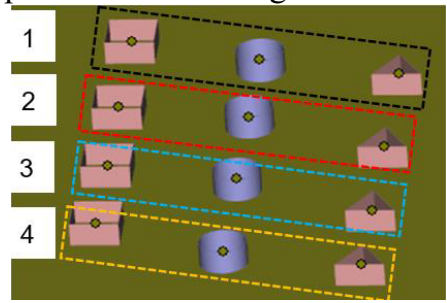

Figure 2. The model of the specimens.

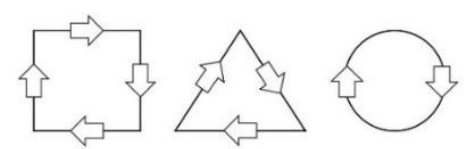

Figure 3. The scan path of the laser.

\subsection{Experimental process}

The date of the intensity of the molten pool and the area of the molten were collected and analyzed, the wall-thickness of the specimens were measured by using a vernier caliper, the roundness tolerance and the flatness tolerance of the specimens were measured by using a three-coordinate measuring machine (Global, Hexagon, Sweden). The simulation model shown in Fig.4 was built for the experiment to simulate the temperature field during the process. The size of the specimens is $1.6 \mathrm{~mm}$ $\times 0.16 \mathrm{~mm} \times 0.12 \mathrm{~mm}$. The grid is a hexahedral grid, and the size is $0.04 \mathrm{~mm} \times 0.04 \mathrm{~mm} \times 0.04 \mathrm{~mm}$. The materials of the substrate and that the specimens is same, the substrate is divided into upper and lower parts. The lower part adopts a $0.4 \mathrm{~mm}$ larger grid and the upper part adopts a free grid to realize tetrahedral transition [11].

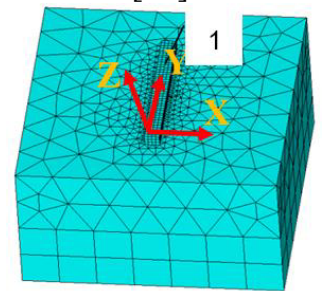

Figure 4. Finite element model.

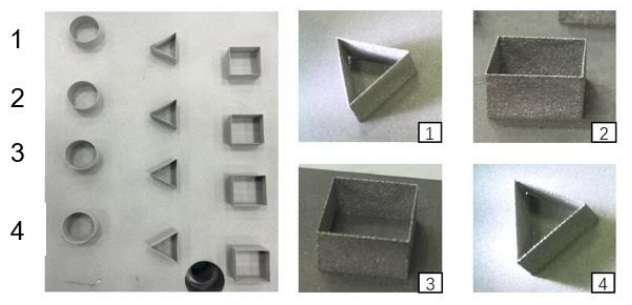

Figure 5. Fabricated specimens.

\section{Results}

The fabricated specimens shown as Fig.5. The square and triangular had a little deformation at the center of the wall, and the corners of the specimens were separated from the substrate. The circular specimens were of good quality and have no deformation.

\subsection{The wall-thickness of the specimens}


The wall-thickness of the specimens were measured at ten different positions selected randomly and averaged, the result was shown as Fig.6. The wall-thickness increases with the increasing of the laser power while the linear energy density is same. The wall-thickness value of the circular specimens is maximum and the wall-thickness value of the triangular specimens is minimum. Then simulated the temperature field of the molten pool, at the position of $\mathrm{Y}=0$, the temperature curves with different process parameters are shown in Fig.7. The temperature higher than liquidus temperature is the molten pool, it shows that the highest temperature of the molten pool gradually increases as the laser power is increasing from $120 \mathrm{~W}$ to $300 \mathrm{~W}$ and the width of the molten pool has the same tendency.

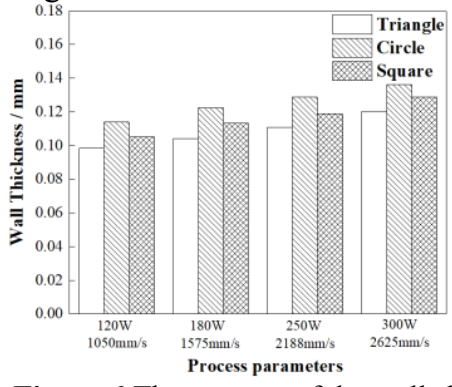

Figure 6 The average of the wall-thickness.

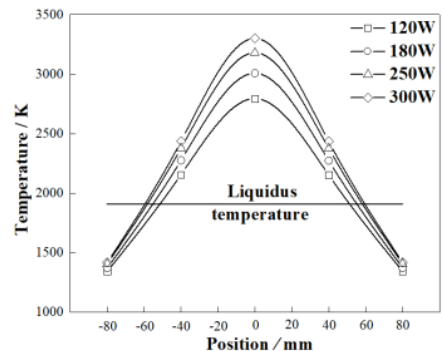

Figure 7 Temperature curves.

The data of the molten pool collected by QM-Meltpool is shown in Fig.8. As the power increasing, the area of the molten pool gradually increases shown as Fig.8(a) with then same tendency of the result of the wall-thickness (Fig.6) and the width simulation result of the molten pool (Fig.7). As the laser power increases, the temperature around the laser spot increases. There is more powder melted. The width of the molten pool and the wall-thickness of the specimens increase. When the linear energy density is same, the intensity of the molten pool increases due to the increasing of the area of the molten pool, shown as Fig.8(b).
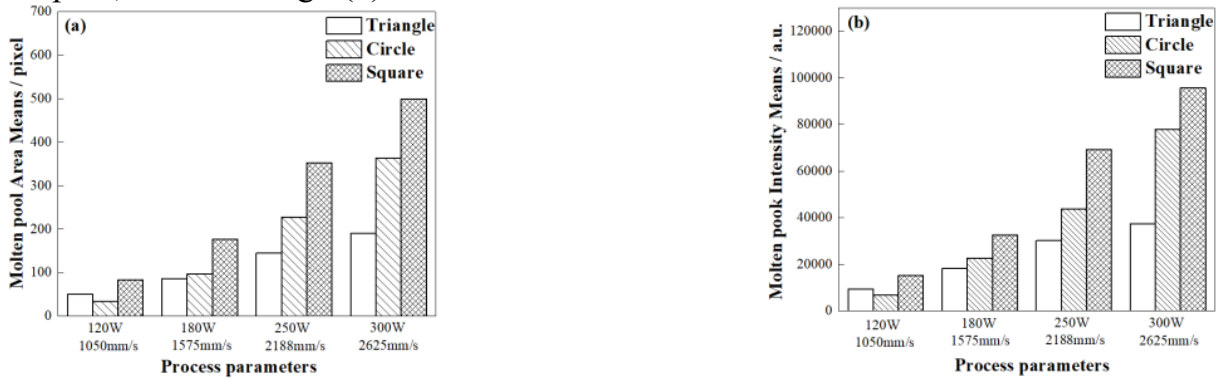

Figure 8 The data of the molten pool (a) the area of the molten pool and (b) the intensity of the molten pool.

\subsection{The deformation of the specimens}

The measurement results of the geometric tolerance are shown in Fig. 9. Under the condition that the online energy density is the same, the laser power increased from $120 \mathrm{~W}$ to $300 \mathrm{~W}$, and the roundness average value shows a downward trend, indicating that the deformation of the test piece is reduced (Fig. 9(a)); likewise, the flatness of the triangular and square specimens also showed a downward trend (Fig. 9(b)). The above results show that under the condition of the same online energy density, the increase of laser power and scanning rate can effectively reduce the deformation degree of the test piece and improve the quality of the formed part. 

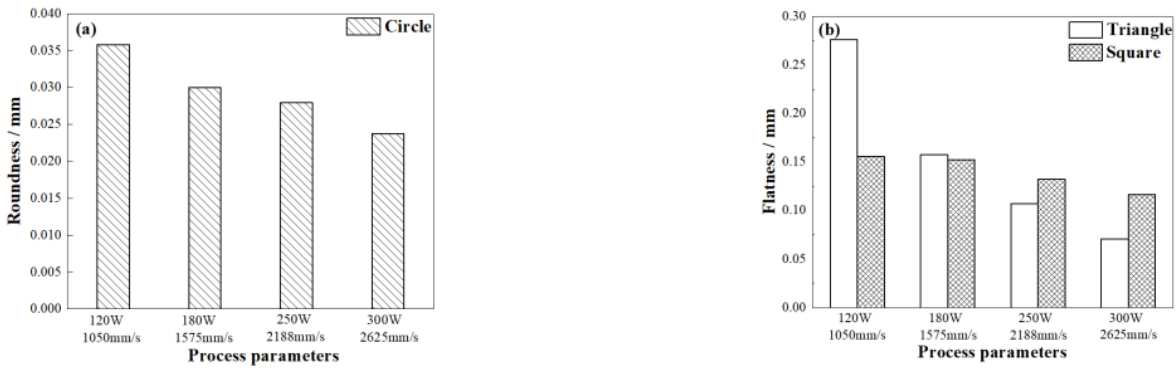

Figure 9 Shape tolerance measurement results (a) roundness tolerance and (b) flatness tolerance.

\section{Discuss}

The powder solidified rapidly after being melted, the wetting quality between the molten pool and the substrate, the solidification rate of the molten pool and the cooling rate of the specimens have a significant influence on the quality of the specimens, and all these influenced by the temperature of the molten pool.

\subsection{The deformation of the specimens}

In the SLM process, the law of the temperature changing at different position in the molten pool is same, taking a node in the second layer of this model randomly as $(0,0)$, the temperature change process of this node with different process parameters as the laser spot scanning along the Y-axis shown as Fig.10.

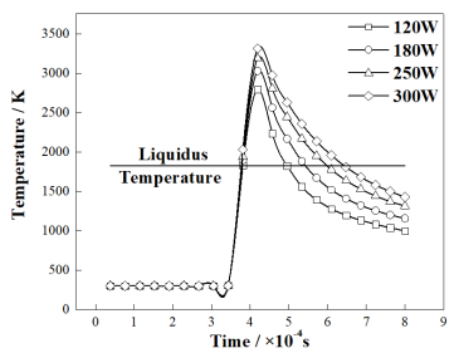

Figure 10 Temperature curves.

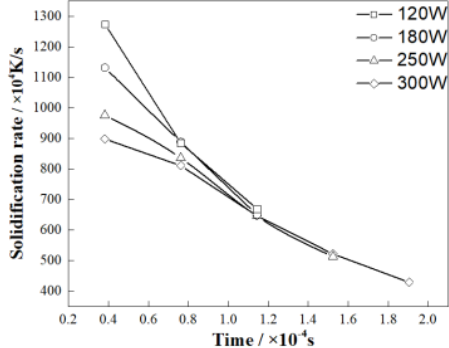

Figure 11 Simulation results about the solidification rate. As the increasing of the laser power, the highest temperature of the $(0,0)$ increases from the 2794 $\mathrm{K}$ to $3312 \mathrm{~K}$ as the laser power is increasing from $120 \mathrm{~W}$ to $300 \mathrm{~W}$. The solidification time of droplet can be calculated using Eq. (2), the equation is as follow[12]:

$$
\tau_{\text {soli }}=2\left(\frac{a^{2}}{3 \alpha}\right) \ln \left(\frac{T_{0}-T_{t}}{T_{f}-T_{t}}\right)
$$

where $a$ is the size of the initial droplet, $\alpha$ is the material thermal diffusion coefficient, $T_{0}$ is the temperature of the initial droplet, $T_{f}$ is the liquidus temperature of material, $T_{t}$ is the temperature of the substrate, and $\tau_{\text {soli }}$ is the solidification time of droplet.

According to the Eq. (2), the solidification time of the molten pool increases with the increasing of the initial size of the droplet and the initial temperature of the droplet. The solidification time of the molten pool increases with the increasing of the laser power due the increasing of the size of the initial droplet and the temperature of the initial droplet (Fig.6 and Fig.10). The longer solidification time is good for spreading and wetting with the last layer and can avoid the balling of the droplet due to the short solidification time as long as possible, which is good to improving the quality of the powder layer, and reducing the remelting phenomenon. This can reduce the thermal stress caused by remelting and reduce the possibility of the deformation. 


\subsection{The solidification shrinkage}

Due to the thermal expansion of the material, volume condensation occurs during the solidification of the droplet, and adjacent regions of the droplet will produce different degrees of shrinkage due to different solidification rates. The shrinkage of the specimens can be calculated using Eq. (3).

$$
\partial l=\delta \times l \times \partial t
$$

where $\delta$ is the materials thermal expansion coefficient, $l$ is the size of the specimens and $\partial t$ is the amount of change in temperature per unit time. In this experiment, the same material is used, and the thermal expansion coefficient is same; the shape of the specimens is same. The amount of shrinkage is related to the solidification rate. In this solidification process of the droplet, the faster the solidification rate, the larger amount of shrinkage will be, and more obviously of the deformation will be. Taking the derivation with respect to the temperature, the results of the solidification rate is shown as Fig.11. The solidification rate shows obvious decreasing as the laser power increasing from $120 \mathrm{~W}$ to $300 \mathrm{~W}$. When the laser power is higher, the amount of the shrinkage $\partial l$ is smaller, the tendency is same as the change rules of deformation shown as Fig.6.

The specimens rapidly cooled after the droplet solidified. The temperature at different positions was uneven due to the different cooling rate, resulting in the temperature gradient. The difference in cooling rate between adjacent regions caused different degrees of shrinkage, and the portion with a large amount of shrinkage was constrained by the portion with a small amount of shrinkage, which caused by uneven temperature distribution and was also called thermal stress. As the scanning of the laser spot, the stress accumulated. Once the stress exceeded the yield strength of the materials, the deformations of the specimens appeared, even the cracks. The thermal stress can be calculated using Eq. (4).

$$
\begin{gathered}
\sigma_{x}=\frac{\delta E \Delta T_{x}}{1-2 u} \\
\sigma_{y}=\frac{\delta E \Delta T_{y}}{1-2 u} \\
\sigma_{z}=\frac{\delta E \Delta T_{z}}{1-2 u}
\end{gathered}
$$

where $\sigma_{x}$ is the thermal stress in the direction of X, $\sigma_{y}$ is the thermal stress in the direction of $\mathrm{Y}, \sigma_{z}$ is the thermal stress in the direction of $\mathrm{Z}, \delta$ is the materials thermal expansion coefficient, $E$ is the materials modulus of elasticity, $u$ is the Poisson's ratio of materials, $\Delta T_{x}$ is the temperature gradient in the direction of $\mathrm{X}, \Delta T_{y}$ is the temperature gradient in the direction of $\mathrm{Y}$ and $\Delta T_{z}$ is the temperature gradient in the direction of $\mathrm{Z}$. The deformation caused by the thermal stress can by calculated using Eq. (5).

$$
\begin{aligned}
\varepsilon_{x} & =\frac{1}{E}\left[\sigma_{x}-u\left(\sigma_{y}+\sigma_{z}\right)\right]+\delta \Delta T_{x} \\
\varepsilon_{y} & =\frac{1}{E}\left[\sigma_{y}-u\left(\sigma_{x}+\sigma_{z}\right)\right]+\delta \Delta T_{y} \\
\varepsilon_{z} & =\frac{1}{E}\left[\sigma_{z}-u\left(\sigma_{x}+\sigma_{y}\right)\right]+\delta \Delta T_{z}
\end{aligned}
$$

where $\varepsilon_{x}$ is the deformation in the direction of $\mathrm{X}, \varepsilon_{y}$ is the deformation in the direction of $\mathrm{Y}$ and $\varepsilon_{z}$ is the deformation in the direction of Z. Then combined the Eq. (4) with the Eq. (5), the deformation was shown as Eq. (6).

$$
\begin{aligned}
\varepsilon_{x} & =2 \delta \Delta T_{x} \\
\varepsilon_{y} & =2 \delta \Delta T_{y} \\
\varepsilon_{z} & =2 \delta \Delta T_{z}
\end{aligned}
$$

It is known that the thermal stress was decided by the temperature gradient. The higher the temperature gradient, the larger the deformation amount caused by the thermal stress will be.

The molten pool simulation results are shown as Fig.12. The size of the molten pool increased obviously with the increase of the laser power. 


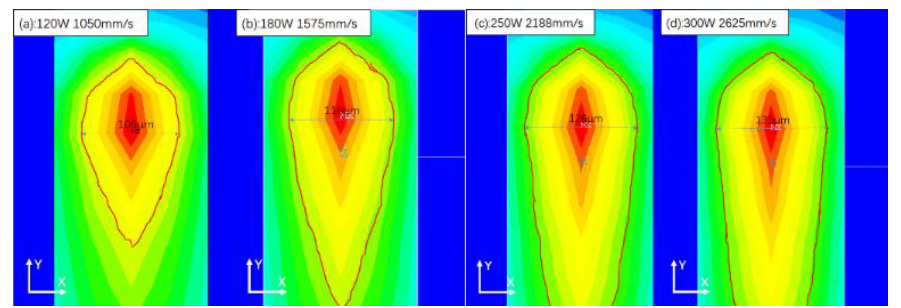

Figure 12 The dimensions of the molten pool, (a)120W,1050mm/s;(b)180W,1575mm/s;(c)250W,2188mm/s and (d) $300 \mathrm{~W}, 2625 \mathrm{~mm} / \mathrm{s}$.

When the laser spot scanning along with the direction of $Y$, taking the position at $(0,0)$ with the highest temperature, then calculated the temperature gradient (TG) along X-direction and the TG along Y-direction respectively at the position of $(40,0)$ and $(0,-40)$, the results are shown as Fig.13.
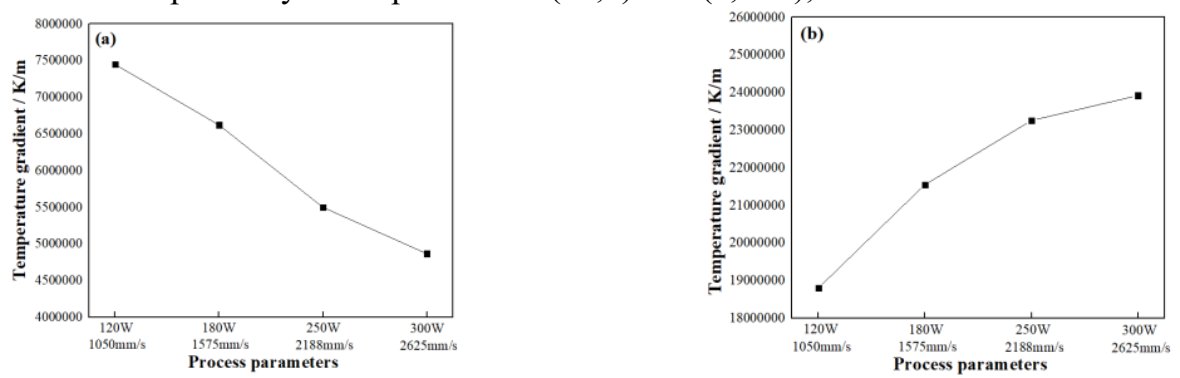

Figure 13 Influence of process parameters on the TG of the point $(0,-40)$ and $(40,0)$ as the laser beam reach the point $(0,0)$, (a)the TG of the point $(0,-40)$ along Y-direction and (b) the TG of the point $(40,0)$ along X-direction.

When the linear energy density is same, $\Delta T_{y}$ decreases with the increasing of the laser power shown as Fig.13 (a) and $\Delta T_{y}$ shows the contrary tendency shown as Fig.13 (b). This means that $\varepsilon_{y}$ caused by $\Delta T_{y}$ was smaller with the increase of the laser power as the linear energy density is same and $\varepsilon_{x}$ caused by $\Delta T_{x}$ shown the contrary tendency. The $\varepsilon_{x}$ on both side have equal value and opposite directions, and the deformation caused by $\Delta T_{x}$ at different side cancelled each other out. The $\Delta T_{y}$ has the significant influence on the deformation of the specimens and the deformation is smaller with higher laser power when the linear energy density is same.

\section{Conclusions}

In this work, the Ti6Al4V thin-wall specimens were fabricated by Selective Laser Melting (SLM) using various process parameters. The laser power had a significant influence on the deformation of the specimens. The main conclusions are summarized as follows:

(1) When the linear energy density is same, the solidification rate of the molten pool decreases with the increasing of the laser power, the shrinkage during the droplet solidification process is smaller with a higher laser power. The $\Delta T_{y}$ decreases with the increase of the laser power and the $\varepsilon_{y}$ caused by the $\Delta T_{y}$ is smaller with a higher laser power and the $\Delta T_{y}$ has the significant influence on the deformation of the specimens. When the laser power increased from $120 \mathrm{~W}$ to $300 \mathrm{~W}$, the roundness of the circular specimen reduced from $0.034 \mathrm{~mm}$ to $0.024 \mathrm{~mm}$; the flatness of the triangular specimens and the square specimens reduced from $0.27 \mathrm{~mm}$ and $0.16 \mathrm{~mm}$ to $0.07 \mathrm{~mm}$ and $0.11 \mathrm{~mm}$ respectively.

(2) When the linear energy density is same, the intensity of the meltpool and the area of the molten pool increase with the increasing of the laser power.

(3) When the linear energy density is same, the solidification time of the molten pool increases with the increasing of the laser power, so as the temperature of meltpool, which is good for molten pool wetting with the substrate, improving the quality of the powder layer and reducing the balling phenomenon. 


\section{References}

1. Z.T. Wu, X.T. Hu, T.D. Song, Multiaxial fatigue life prediction for titanium alloy TC4 under proportional and nonproportional loading. Int. J. Fatigue,59(3):170-175(2014).

2. D. Herzog, V. Seyda, E. Wycisk, C. Emmelmann, Additive Manufacturing of Metals, Acta Mater.117:371-392(2016).

3. L.S. Bertol, W.K. Júnior, F.P.D. Silva, C. Aumund-Kopp. Medical design: Direct metal laser sintering of Ti-6Al-4V, Mater. Des. 31(8) : 3982-3988(2010).

4. L.Thijs, F. Verhaeghe, T. Craeghs, J.V. Humbeeck, J.P. Kruth. A study of the microstructural evolution during selective laser melting of Ti-6Al-4V, Acta Mater. 58(9):3303-3312(2010).

5. B.Vrancken, V.Cain, R.Knutsen, J.V. Humbeeck. Residual stress via the contour method in compact tension specimens produced via selective laser melting, Scripta Mater. 87(87):29-32(2014).

6. Additive manufacturing of high-strength aluminum components, Annual report fraunhofer institute for technology(2010).

7. D. Buchbinder. Generative fertigung von Aluminiumbauteilen für die Serienproduktion, AluGenerativ Abschlussbericht.

8. S.Siddique, M.Imran, E.Wycisk, C.Emmelmann, F.Walther. Influence of process-induced microstructure and imperfections on mechanical properties of AlSi12 processed by selective laser melting, J. Mater. Process Tech. 221:205-213(2015).

9. M.Karg, B. Ahuja, S. Kuryntsev, A. Gorunov, M.Schmidt. Processability of high strength Aluminium-Copper alloys AW-2022 and 2024 by Laser Beam Melting in Powder Bed. International Solid Freeform Symposium(2014).

10. K.Zhang, T.T.Liu, C.D.Zhang, W.H.liao. Study on Deformation Behavior in Selective Laser Melting Based on the Analysis of the Melt Pool Data. Chinese J. Lasers. 42(9): 0903007(2015).

11. D.N.Chen, T.T.Liu, W.H.liao, C.D.Zhang, K.Zhang. Temperature Field During Selective Laser Melting of Metal Powder Under Different Scanning Strategies, Chinese J. Lasers. 43(4):0403003(2016).

12. F. Gao, A.A. Sonin, Precise Deposition of Molten Microdrops: The Physics of Digital Microfabrication, Proc. R. Soc. London. Ser. A. 444 :533-554 (1994). 